FARIA RT; COLOMBO RC; HOSHINO RT. 2015. Oncidium sarcodes x Oncidium Aloha 'Iwanaga': nova opção de híbrido de Oncidium. Horticultura Brasileira 33: 131-134. DOI - http://dx.doi.org/10.1590/S0102-053620150000100021

\title{
Oncidium sarcodes x Oncidium Aloha 'Iwanaga': nova opção de híbrido de Oncidium
}

\author{
Ricardo T Faria; Ronan C Colombo; Rodrigo T Hoshino \\ UEL, Depto. de Agronomia, C. Postal 10.011, 86057-970 Londrina-PR; faria@uel.br (bolsista de produtividade CNPq)
}

\begin{abstract}
RESUMO
O gênero Oncidium apresenta cerca de 315 espécies e centenas de híbridos. O melhoramento genético com espécies nativas, de potencial ornamental, é uma importante estratégia para garantir melhor adaptabilidade das cultivares obtidas às condições brasileiras de cultivo. Dentre elas, a espécie brasileira Oncidium sarcodes, se destaca por longas hastes com flores grandes e amarelas, com máculas marrons. Objetivou-se nesse trabalho caracterizar progênies obtidas da hibridação entre oncidiums, de modo a selecionar uma potencial cultivar. Plantas selecionadas de $O$. sarcodes foram utilizadas como parental feminino e $O$. Aloha 'Iwanaga' como parental masculino. Para o cruzamento utilizou-se polinização manual controlada e as sementes obtidas desse cruzamento foram germinadas in vitro. Após o crescimento in vitro e aclimatização das plântulas em casa de vegetação, as mesmas foram transplantadas para vasos com volume de $1000 \mathrm{~mL}$ utilizando como substrato casca de pinus e fibra de coco, na proporção de 1:1 (v/v). Para a descrição morfológica dos híbridos selecionou-se 10 progênies, nas quais foram avaliadas as características fitométricas da parte vegetativa e das inflorescências. $\mathrm{O}$ florescimento dos híbridos ocorreu aos 48 meses após a germinação, entre os meses de dezembro a janeiro, com flores durando entre 25 e 30 dias. $\mathrm{O}$ cruzamento trouxe às flores maior equilíbrio e simetria entre os verticilos florais. As sépalas e as pétalas apresentam coloração amarelo ouro com máculas marrons, com limite bem determinado entre as cores.
\end{abstract}

Palavras-chave: Floricultura, melhoramento, orquídea, cruzamento, polinização controlada.

\begin{abstract}
Oncidium sarcodes x Oncidium Aloha 'Iwanaga': new option of Oncidium hybrid
\end{abstract}

Oncidium genus has about 315 species and hundreds of hybrids. Breeding of native species with ornamental potential is an important strategy to ensure a better adaptability of cultivars to Brazilian conditions. Among them, the Brazilian species Oncidium sarcodes stands out for long stems with large yellow flowers with brown stains. The aim of this work was to characterize the progenies resulting from the oncidiums hybridization in order to select a potential cultivar. Selected $O$. sarcodes plants were used as the female parental and O. Aloha 'Iwanaga' as the male parental. Pollination was manually controlled and the obtained seeds were germinated in vitro. After seedling establishment in vitro and acclimatization in the greenhouse, they were transplanted to plastic pots with volume of $1000 \mathrm{~mL}$ using as substrate pine bark and coconut fiber 1:1 (v/v). For the morphological description of the hybrids, 10 progenies were selected to evaluate the characteristics of vegetative development and inflorescences. Flowering occurred 48 months after germination, from December to January, flowers with durability of 25 to 30 days. The crossing brought flowers to a greater balance and symmetry between the verticils. The sepals and petals have yellow gold color with brown spots, with well-defined limits between the colors.

Keywords: Floriculture, breeding, orchid, crossing, controlled pollination.

\section{(Recebido para publicação em 6 de junho de 2014; aceito em 29 de agosto de 2014) (Received on June 6, 2014; accepted on August 29, 2014)}

\begin{abstract}
A comercialização dos produtos da floricultura em supermercados, feiras e eventos, tem criado nos produtores a necessidade de buscar e produzir novas variedades e híbridos; reflexo da procura por novidades e qualidade, pelos consumidores (Takane et al., 2010). Assim, considerando estes aspectos e tendo em perspectiva a grande diversidade genética de espécies com fins ornamentais que são nativas do Brasil, uma estratégia para alavancar o setor nacional e torná-lo autossuficiente é o estabelecimento de programas brasilei-
\end{abstract}

ros de melhoramento genético de flores e plantas ornamentais (Cardoso, 2013).

Para isso, é necessário que sejam feitos maiores investimentos em prospecção e biologia reprodutiva das principais espécies cultivadas no país, de modo a desenvolver cultivares com padrão de excelência similar e até mesmo superior ao das cultivares estrangeiras (Cardoso, 2013). Além disso, o uso de espécies nativas, nesses programas de melhoramento genético, é uma estratégia para assegurar maior adaptabilidade às condições climáticas e de cultivo no Brasil.
No entanto, para a seleção de plantas ornamentais de qualidade superior, devem-se considerar as características ornamentais como coloração, tamanho e proporcionalidade das flores, além do vigor vegetativo; e as características agronômicas como o crescimento rápido e vigoroso, florescimento precoce, durabilidade das flores, resposta à adubação, facilidade de cultivo, resistência a pragas e doenças; a adaptabilidade às diferentes regiões de cultivo e o aspecto inovador da cultivar, que a torna interessante comercialmente (Cardoso, 2010). 
O gênero Oncidium, pertence à tribo Maxillarieae e à subtribo Oncidiinae; no Brasil são encontradas 94 espécies nativas, das 315 espécies distribuídas nesse gênero. As diferentes espécies apresentam uma grande variação morfológica e ecológica entre si, crescem desde o nível do mar até altitudes bastante elevadas, 4.000 metros. A maioria das espécies é epífita, algumas são terrícolas e há outras rupícolas (Ferrarezi et al., 2007).

Oncidium sarcodes, planta epífita é encontrada em alguns estados do sudeste e sul do Brasil. Apresenta crescimento simpodial, pseudobulbos verde-escuros, 1-3 foliados, apresenta inflorescência surgindo da base dos pseudobulbos, formada por mais de 150 flores, descendente, panícula, com aproximadamente $200 \mathrm{~cm}$ de comprimento. Flores com sépala dorsal curvada para frente, castanho-avermelhada, sépalas laterais amareladas e pétalas laterais castanho-avermelhadas, labelo trilobado e amarelo (Ferrarezi et al., 2007).

Oncidium Aloha 'Iwanaga' é um híbrido obtido a partir do cruzamento de Oncidium Goldiana x Oncidium Star Wars. Essa cultivar, como outras do gênero, é classificada no grupo das orquídeas "sprays", pois apresenta numerosas flores amarelo-ouro em haste mais compacta, formando um "spray" dourado. As flores apresentam labelo trilobado, que se destaca pelas suas maiores proporções em relação às demais pétalas e sépalas (Colibri Orquídeas, 2014; Vorobik Botanical Art, 2014).

Assim, objetivou-se nesse trabalho a hibridação desses genótipos visando caracterizar as progênies e selecionar uma potencial cultivar de Oncidium.

\section{MÉTODO DE MELHORA- MENTO}

Plantas sadias e vigorosas, cultivadas no orquidário da Universidade Estadual de Londrina, com elevado número de flores foram utilizadas como matrizes para os cruzamentos entre Oncidium sarcodes e Oncidium Aloha 'Iwanaga'.

O processo de hibridação ocorreu utilizando polinização manual controla- da; políneas da planta doadora (O. Aloha 'Iwanaga') foram coletadas de flores recém-abertas (48 horas após a antese) e transferidas para o estigma de flores recém-abertas (24 a 48 horas após a antese) da planta receptora (O. sarcodes). Nove meses após o cruzamento foram obtidas as cápsulas (frutos) contendo as sementes, as quais foram germinadas in vitro em meio de cultura MS (Murashige \& Skoog, 1962), com a metade da concentração dos macronutrientes. A base dos meios foi constituída por $30 \mathrm{~g} / \mathrm{L}$ de sacarose, $1 \mathrm{~g} / \mathrm{L}$ de carvão ativado e 7,5 $\mathrm{g} / \mathrm{L}$ de ágar; o $\mathrm{pH}$ foi ajustado para 5,8 antes da adição do ágar e autoclavagem. As plântulas obtidas no processo de germinação foram subcultivadas em mesmo meio de cultura até atingirem idade para aclimatização, nove meses após a germinação, com aproximadamente oito centímetros de altura. Os frascos permaneceram em sala de crescimento sob fotoperíodo de 16 horas de luz

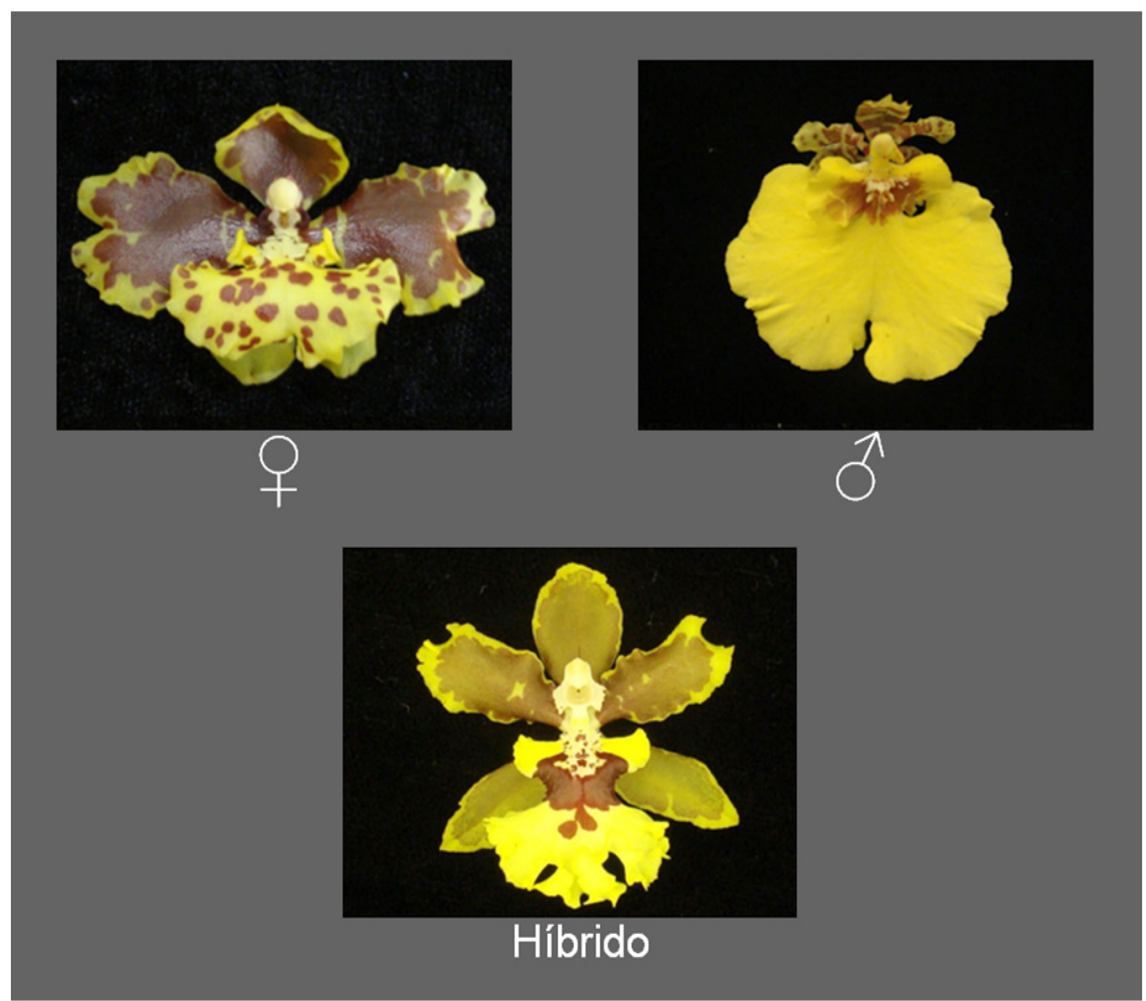

Figura 1. Flor de Oncidium sarcodes (parental feminino, imagem superior esquerda), Oncidium Aloha 'Iwanaga' (parental masculino, imagem superior direita) e híbrido (imagem inferior central) obtida a partir do cruzamento artificial (flower of Oncidium sarcodes (female parental, left and higher image), Oncidium Aloha 'Iwanaga' (male parental, right and higher image) and hybrid (below and center image) obtained from artificial crossing). Londrina, UEL, 2014.

Tabela 1. Caracteres morfológicos vegetativos de Oncidium sarcodes x Oncidium Aloha 'Iwanaga'; comprimento (CPB) e largura (LPB) do pseudobulbo, comprimento (CFO) e largura (LFO) da folha \{vegetative morphological traits of Oncidium sarcodes x Oncidium Aloha 'Iwanaga'; length (CPB) and width (LPB) of the pseudobulb, length (CFO) and width (LFO) of leaves . Londrina, UEL, 2014.

\begin{tabular}{lcccc}
\hline Variável & CPB (cm) & LPB (cm) & CFO (cm) & LFO (cm) \\
\hline Amplitude & $12,0-18,0$ & $3,5-4,0$ & $30,0-42,0$ & $4,1-5,1$ \\
Média* & 14,3 & 3,8 & 35,3 & 4,5 \\
Erro padrão & 0,997 & 0,102 & 1,706 & 0,141 \\
\hline
\end{tabular}

*Média obtida a partir de 120 flores observadas em 10 progênies (average obtained from 120 flowers observed in 10 progenies). 
Tabela 2. Caracteres morfológicos de inflorescências e flores de Oncidium sarcodes $\mathrm{x}$ Oncidium Aloha 'Iwanaga'; comprimento da haste floral (CHF), número de flores por haste (NFL), comprimento da flor (CFL), largura da flor (LFL), comprimento do labelo (CLA) e largura do labelo (LLA) \{morphological characters of inflorescences and flowers of Oncidium sarcodes x Oncidium Aloha 'Iwanaga'; flower stem length (CHF), number of flowers per stem (NFL), flower length (CFL), width of flower (LFL), length of the lip (CLA) and width of the lip (LLA)\}. Londrina, UEL, 2014.

\begin{tabular}{lcccccc}
\hline Variável & $\begin{array}{c}\text { CHF } \\
(\mathbf{c m})\end{array}$ & $\mathbf{N F L}$ & $\begin{array}{c}\text { CFL } \\
\mathbf{( c m )}\end{array}$ & $\begin{array}{c}\text { LFL } \\
(\mathbf{c m})\end{array}$ & $\begin{array}{c}\text { CLA } \\
(\mathbf{c m})\end{array}$ & $\begin{array}{l}\text { LLA } \\
(\mathbf{c m})\end{array}$ \\
\hline Amplitude & $94,0-166,0$ & $32-59$ & $3,5-4,1$ & $3,0-3,5$ & $1,8-2,1$ & $1,6-2,2$ \\
Média* & 121,8 & 48,5 & 3,9 & 3,2 & 1,9 & 1,9 \\
Erro Padrão & 11,256 & 3,939 & 0,095 & 0,076 & 0,042 & 0,086 \\
\hline
\end{tabular}

*Média obtida a partir de 120 flores observadas em 10 progênies (average obtained from 120 flowers observed in 10 progenies).

(lâmpada fluorescente) e temperatura de $25 \pm 2,0^{\circ} \mathrm{C}$.

As plântulas foram aclimatizadas em bandejas de isopor, de $25 \times 25 \mathrm{~cm}$, sem células, contendo aproximadamente 30 plântulas por bandeja, contendo esfagno como substrato. As bandejas foram acondicionadas em estufa agrícola, coberta com plástico, com $60 \%$ de sombreamento, obtido por tela de polipropileno de cor preta. Nessa fase realizou-se adubação foliar mensal,

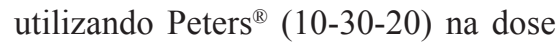
de $3 \mathrm{~g} / \mathrm{L}$.

Após 120 dias em tais condições, as plantas foram transplantadas para vasos plásticos, de coloração preta, com diâmetro de $6 \mathrm{~cm}$ e altura de $8 \mathrm{~cm}$, nos quais permaneceram por seis meses, sendo novamente transplantadas para vasos plásticos, de coloração preta, com diâmetro de $17 \mathrm{~cm}$ e altura de $15 \mathrm{~cm}$. O substrato utilizado foi casca de pinus e fibra de coco (Amafibra ${ }^{\circledR}$, Holambra, Brasil) $(1: 1, \mathrm{v} / \mathrm{v})$. Nestas fases as plantas foram adubadas com Biofert Plus ${ }^{\circledR}(08$ 09-09), $5 \mathrm{~mL} / \mathrm{L}$ de água, a cada 30 dias.

Para a descrição morfológica dos híbridos selecionou-se 10 plantas em que foram avaliadas as características fitométricas da parte vegetativa: comprimento e diâmetro do pseudobulbo e comprimento e largura das folhas; e as características fitométricas da parte reprodutiva: comprimento da haste, comprimento e largura das flores, número de flores por haste. As dimensões dos verticilos florais foram determinadas a partir da mensuração de quatro flores
As sépalas e as pétalas apresentam coloração amarelo ouro com máculas marrons, com limite bem determinado entre as cores, podendo apresentar padrões distintos, em que no primeiro prevalece a coloração marrom em grande parte de superfície, e no segundo as manchas são menores e distribuídas aleatoriamente.

O labelo possui em média comprimento de $1,9 \pm 0,1 \mathrm{~cm}$ e largura $1,9 \pm 0,2$ cm (Tabela 2). 3-lobado com lóbulos laterais pequenos e istmo estreito, margem fortemente ondulada e calo verrugoso denticulado carnoso de coloração clara com pontuações marrom avermelhado. Esta mesma cor está presente no istmo podendo apresentar-se em manchas pequenas nos lóbulos, nos quais pode haver variações entre as tonalidades de amarelo.

O primeiro florescimento do híbrido ocorreu aos 48 meses após a germinação das sementes, entre os meses de dezembro e janeiro; após mais 12 meses, as progênies floresceram novamente na mesma época. A longevidade das flores é cerca de 25-30 dias. Tal período de floração é interessante por coincidir com datas festivas. O híbrido apresenta pseudobulbos e folhas semelhantes a O. Aloha 'Iwanaga', apesar de suas maiores proporções. As inflorescências são longas panículas, herdadas de $O$. sarcodes, entretanto com maior densidade de flores, deixando menos espaços vazios na haste floral. O cruzamento trouxe às flores maior equilíbrio e simetria entre os verticilos florais, combinando o labelo vistoso e exposto de O. Aloha 'Iwanaga', com o tamanho das sépalas e pétalas de $O$. sarcodes (Figura 1).

\section{MANUTENÇÃO E DISTRIBUI- ÇÃO DE PLANTAS}

Plantas resultantes do cruzamento $O$. sarcodes x $O$. Aloha 'Iwanaga' são micropropagadas e mantidas em casa de vegetação pelo Departamento de Agronomia da Universidade Estadual de Londrina, Rodovia Celso Garcia Cid, Pr 445 km 380, Campus Universitário, 
C. Postal 10.011, CEP 86057-970, Londrina-PR.

\section{AGRADECIMENTOS}

À Coordenação de Aperfeiçoamento de Pessoal de Nível Superior (Capes), ao Conselho Nacional de Desenvolvimento Científico e Tecnológico (CNPq) pelo apoio financeiro e ao senhor Geraldo Lopes da Silva, técnico do Orquidário da Universidade Estadual de Londrina.

\section{REFERÊNCIAS}

CARDOSO JC. 2010. Laeliocattleya 'Brazilian Girl Rosa': cultivar de orquídea para cultivo em vaso. Horticultura Brasileira 28: 378-381.

CARDoso JC. 2013. Melhoramento de espécies ornamentais como estratégia para o desenvolvimento e autossuficiência do setor. Horticultura Brasileira 31: 171-171.

COLIBRI ORQUÍDEAS. 2014. Disponível em http://www.colibriorquideas.com/especies/ OncidiumAlohaIwanaga.php

FERRAREZI E; VIEIRA AOS; FARIA RT.
Orquídeas: o gênero Oncidium no Paraná. Londrina: EDUEL, 2007. 120p.

MURASHIGE T; SKOOG F. 1962. A revised medium of rapid growth and bioassay with tobacco tissue cultures. Physiologia Plantarum 15: 473-479.

TAKANE RJ; YANAGISAWA SS; PIVETTA KFL. 2010. Cultivo moderno de orquideas: Cattleya e seus hibridos. Fortaleza: UFC. $179 \mathrm{p}$.

VOROBIK BOTANICALART. 2014. Disponível em http://www.vorobikbotanicalart.com/ Onc\%20Clown\%20det.html 